\title{
Absence of the celiac trunk: definition, classification, MDCT angiographic findings and their probable embryological mechanisms
}

\section{Yi Mao}

Department of Radiology, Daping Hospital, Army Medical University

\section{Wei Tang}

Department of Radiology, Daping Hospital, Army Medical University

\section{Bo Jin}

Department of Radiology, Daping Hospital, University

\section{Lian-Qin Kuang}

Department of Radiology, Daping Hospital, Army Medical University

Jing Zhang

Department of Radiology, Daping Hospital, Army Medical University

Yi Wang ( $\nabla$ ywhxl@qq.com)

Daping Hospital, Army Medical University https://orcid.org/0000-0002-0818-724X

\section{Research article}

Keywords: Celiac artery; Mesenteric artery, superior; Anatomic variation; Classification; Computed tomography angiography

Posted Date: June 20th, 2019

DOI: https://doi.org/10.21203/rs.2.10432/v1

License: (9) This work is licensed under a Creative Commons Attribution 4.0 International License. Read Full License 


\section{Abstract}

Background: The aim of this study was to identify the types and prevalence of absence of the celiac trunk by using multidetector computed tomography (MDCT) angiography, and analyze their probable embryological mechanisms. Methods: A retrospective study was carried out on 2,500 abdominal MDCT angiography images. The absence of the celiac trunk was defined as that the celiac trunk is not exist, more specifically, there is not such an arterial trunk containing at least two major branches of the celiac trunk. Various types of the absence of the celiac trunk were investigated. Results: Of the 2,500 patients, $19(0.76 \%)$ patients were identified as an absence of the celiac trunk. According to its definition and classification, the absence of the celiac trunk could be divided into five types: type I (LGA + CHA + SA + SMA), type II (HM trunk + LGA + SA), type III (SM trunk + LGA + CHA), type IV (GM trunk + CHA + SA), and type $V$ (other type); and these types were observed in 5 patients $(0.20 \%), 9$ patients $(0.36 \%), 3$ patients $(0.12 \%), 0$ patients $(0.00 \%)$ and $2(0.08 \%)$ patients, respectively. There were more examples of the types I and II than of the types III-V $(P=0.004)$. Conclusions: We systematically classified the absence of the celiac trunk based on its MDCT angiography findings. Abnormal interruptions and persistence of the longitudinal anastomosis, and regression of vascular root and emergence of replaced artery could all be the embryological mechanisms of various types of the absence of the celiac trunk.

\section{Background}

The celiac trunk, also referred as the celiac artery or the celiac axis, constitutes a surgically significant splanchnic branch of the abdominal aorta and thus it has been studied thoroughly. The current textbook definition of celiac trunk is an arterial trunk originating from the abdominal aorta to the branching point of the common hepatic artery (CHA) and the splenic artery (SA), with the left gastric artery (LGA) being the first branch also stemming from the arterial trunk, namely the so called its normal trifurcation pattern [1]. This typical trifurcation consists of a true tripod and several false tripods [2]. However, considerable variants on the branching pattern of the celiac trunk have been observed in previous autopsy and radiological studies [2-11].

The absence of the celiac trunk in variants of the celiac trunk is the rarest type, previous studies have defined as that it does not exist for celiac trunk, and specially indicated the CHA, SA and LGA originating separately from the abdominal aorta [2-11]. However, in our daily clinical work, we found that the absence of the celiac trunk is not limited to the CHA, SA and LGA originating separately from the abdominal aorta, it can also occur in other forms of anatomical variant. For example, a hepatomesenteric trunk with LGA and SA arising separately from the abdominal aorta, and a splenomesenteric trunk with LGA and CHA arising separately from the abdominal aorta, in these variants, the celiac trunk also does not exist and they should also be consistent with the absence of the celiac trunk. Therefore, there should be a more accurate definition on the absence of the celiac trunk, and its types need to be further explored.

The aim of this study is to create a more accurate definition on the absence of the celiac trunk, and use multidetector computed tomography (MDCT) angiography to identify its types and prevalence in a large 
study population. We also discuss their clinical implications and the probable embryological mechanisms by which to explain these observed variants.

\section{Methods}

\section{Patients}

Protocols used in this study were approved by the Health Ethics and Research Committee of the authors' hospital, and the requirement for informed patient consent was waived due to the retrospective nature of the study. Between August 2014 and July 2017, a total of 2,862 adult patients underwent abdominal multiphase enhanced MDCT scans and angiography. Data obtained during the arterial phase were used retrospectively to evaluate the anatomy as well as the origination sites of the celiac trunk and its major branches. Exclusion criteria applied were the presence of any condition likely to affect normal vascular anatomy, the MDCT scan or any image reformation that encountered technical deficiency.

\section{MDCT examinations}

Abdominal MDCT was performed on either a 64-row MDCT scanner (GE Healthcare, United States) or a 256-slice MDCT scanner (Philips Healthcare, Cleveland) with a coverage extending from the dome of the diaphragm to the inferior margin of the right kidney or the symphysis pubis. The scan parameters were as follows: collimation of $64 \times 0.625 \mathrm{~mm}$ or $256 \times 0.5 \mathrm{~mm}$ and table speed of 64 or $256 \mathrm{~mm}$ per rotation. The following were applied to all scans: pitch 0.984 , matrix $512 \times 512$, field of view $180-240 \mathrm{~mm}$, tube voltage $120 \mathrm{kV}$, and tube current $300 \mathrm{~mA}$.

Multiphase enhanced MDCT images were obtained in a craniocaudal direction during the hepatic arterial, portal venous, and equilibrium phases. A dual-head power injector was used to administer contrast agent (Ultravist; Bayer Schering Pharma, Berlin, Germany) at $370 \mathrm{mg}$ of iodine per milliliter and $30 \mathrm{~mL}$ of sterile saline $(0.9 \% \mathrm{NaCl})$. The contrast agent and saline solution were injected at $4 \mathrm{~mL} / \mathrm{sec}$ through an $18-$ gauge plastic intravenous catheter placed in an antecubital vein. Contrast agent volumes were delivered at $2 \mathrm{~mL} / \mathrm{kg}$ body weight, and the upper limit of dose was set to $120 \mathrm{~mL}$ for every patient. Images of the hepatic arterial, portal venous and equilibrium phases were acquired respectively at 20,50 and $120 \mathrm{~s}$ after completion of the contrast medium administration.

\section{Image analysis}

For the purposes of this study, only the data obtained during the arterial phase were downloaded onto an off-line workstation (ADW 4.3; General Electric Healthcare, Milwaukee, USA) for image post-processing and analysis. We used multiplanar reformation (MPR) in three spatial planes and 3-D reformation using volume rendering (VR) and maximum intensity projection (MIP). Images were reformatted and analyzed by three abdominal radiologists with 6,16 , and 25 years of experience, respectively. The radiologists independently assessed each scan with respect to origination sites and the anatomy of the celiac trunk 
and its major branches. In cases with discrepancy, the images were reviewed again, with careful correlation among the radiologists. The anatomy was thereby determined by majority opinion. For the study of variant patterns of the absence of the celiac trunk, we developed a classification (Table 1). Statistical analysis was done with the Statistical Package for the Social Sciences version 22.0 (SPSS, Chicago, IL, USA).

\section{Definition and classification for absence of the celiac trunk}

We analyzed the variant patterns of the absence of the celiac trunk according to the origins for the five major arteries-the celiac trunk, superior mesenteric artery (SMA), LGA, CHA, and SA-with adherence to our definitions of the celiac trunk and the absence of the celiac trunk. We defined the celiac trunk as an arterial trunk containing at least two of its major branches irrespective of its origin and anatomic course which was described in our previous study [1], and the absence of the celiac trunk as that the celiac trunk is not exist, more specifically, there is not such an arterial trunk containing at least two major branches of the celiac trunk.

With the definitions of the celiac trunk and the absence of the celiac trunk, various variant types of the absence of the celiac trunk can be objectively described. In order to comprehensively describe results of a systematic analysis of variant patterns of the absence of the celiac trunk, we developed a new classification. According to the origination sites of the celiac trunk and its major branches, the absence of the celiac trunk can be theoretically divided into five types (Table 1). By integrating data obtained from the analysis of MDCT angiographic images, we classified variants of the celiac trunk and its major branches in the absence of the celiac trunk. Finally, we suggest hypothetical embryological mechanisms by which to explain the observed variants.

\section{Results}

A total of 362 patients were excluded from our study; 341 of them had a pathological condition affecting normal vascular anatomy, twenty-one MDCT examinations were considered technically inadequate, including insufficient arterial contrast enhancement (5 patients), motion artifact (3 patients) and postprocessing deficiency (13 patients), which caused difficulty in identifying the anatomical origins of the celiac trunk or its major branches. Then, a total of 2,500 patients, 1,811 men and 689 women, were included once each in the study, with an age range of 18 to 82 years (mean age, $50.4 \pm 13.8$ years).

\section{Incidence of absence of the celiac trunk}

A total of 2,243 (89.72\%) of the 2,500 patients had a normal aortic origin of celiac trunk and its major branches. Variants of celiac trunk were observed in $257(10.28 \%)$ of the patients. The absence of the celiac trunk was observed in $19(0.76 \%)$ patients in these celiac trunk variants. Among them, 15 were males $(0.83 \%, 15 / 1,811)$ and 4 were females $(0.58 \%, 4 / 689)(P=0.524)$, with male and female ages ranging from 34 to 79 years (mean age, $52.87 \pm 11.42$ years) and 41 to 58 years (mean age, $50.25 \pm 7.18$ years) $(P=0.589)$, respectively. 


\section{Types and percentages of absence of the celiac trunk}

Table 2 shows the percentages for various types of absence of the celiac trunk. According to its variant patterns, the absence of the celiac trunk can be classified into five types. There were more examples of the type I ( 5 patients) and type II (9 patients) than of the type III (3 patients), type IV ( 0 patient) and type V (2 patients) $(P=0.004)$.

Type I (LGA + CHA + SA + SMA), is the classical and most motioned type of absence of the celiac trunk, with the LGA, the CHA, the SA and the SMA originating separately from the abdominal aorta (Fig. 1a). It was found in $26.31 \%(5 / 19)$ in the patients with absence of the celiac trunk and in $0.20 \%(5 / 2,500)$ in all patients studied, was the second most common type.

Type II (HM trunk + LGA + SA), a hepatomesenteric trunk with the LGA and the SA arising separately from the abdominal aorta (Fig. 1b), was found in $47.36 \%$ (9/19) in the patients with absence of the celiac trunk, and $0.36 \%(9 / 2,500)$ in all patients studied. It was the most frequent type observed.

Type III (SM trunk + LGA + CHA), a splenomesenteric trunk with the LGA and the CHA arising separately from the abdominal aorta (Fig. 1c), was found in $15.79 \%(3 / 19)$ in the patients with absence of the celiac trunk, and $0.12 \%(3 / 2,500)$ in all patients studied.

Type IV (GM trunk + CHA + SA), which is a gastromesenteric trunk with the CHA and the SA arising separately from the abdominal aorta, but this type was not found in our study.

Type V (other type), that is, any other variant that meets the definition of the absence of the celiac trunk, was found in $10.53 \%(2 / 19)$ in the patients with absence of the celiac trunk, and $0.08 \%(2 / 2,500)$ in all patients studied. One patient was found to be the LGA arising directly from the abdominal aorta, with the $\mathrm{CHA}$ and the SA originating from the SMA at two different sites (Fig. 1d). Another patient was observed to be a SM trunk with the CHA arising from the abdominal aorta, but the LGA originating unusually from the proper hepatic artery (Fig. 1e).

\section{Concomitant other branch variants of absence of the celiac trunk}

Concomitant variants of other branches in the absence of the celiac trunk included 1 patient with a gastroduodenal artery arising from the SA (Fig. 1a), 1 with the left hepatic artery arising from the LGA (Fig. 1d), and 1 with an arc of Bühler (AOB) communicating with the CHA and the SMA (Fig. 1f). The LGA, $\mathrm{CHA}, \mathrm{SA}$ and SMA of the remaining 16 patients with absence of the celiac trunk were not found to be associated with variant in their main branches.

\section{Other variants of celiac trunk}

In the 2,500 patients, six other types of variants in the celiac trunk and SMA were also observed in a total of $238(9.52 \%)$ of these patients. The most common variants were HM trunk + GS trunk and celiomesenteric trunk, which were found in $103(4.12 \%)$ and $85(3.4 \%)$ of the patients studied, 
respectively. In addition, SM trunk + HG trunk, GS trunk + CHA + SMA, HS trunk + LGA + SMA and HG trunk + SA + SMA were observed in $27(1.08 \%), 13(0.52 \%), 7(0.28 \%)$ and $3(0.12 \%)$ patients, respectively.

\section{Discussion}

The celiac trunk usually divides into three branches-the LGA, CHA and SA. However, many variant patterns of the celiac trunk have been described. In a review of the literature, on 2,141 cadavers, $87.6 \%$ of celiac trunks had the classical trifurcation, $12.2 \%$ presented an incomplete bifurcated celiac trunk and $0.2 \%$ was absent [5]. On a large series of patients $(10,750$ patients from 19 studies), the typical three branched celiac trunk (complete celiac trunk) occurred in $90.70 \%$ of patients, the incomplete celiac trunk in $6.09 \%$ of patients, and the absence of the celiac trunk only in $0.19 \%$ of patients [6]. The absence of celiac trunk was extremely rare, existing classifications were rarely described excepting some case reports $[2,4-6,12]$.

There have been many attempts to classify the different branching patterns of the celiac trunk. Lipshutz [7] seems to have been the first and suggested a classification of the celiac trunk into four types. Adachi [8] presented a more detailed classification, whereas two other commonly used classifications were suggested by Morita [9] and Michels [10]. The first classification system that included an absent celiac trunk as a morphological type was that of Morita. Regrettably, Morita considered the possibility of such a variant, but none was described. Recently, Marco-Clement et al [11] created a new and simple classification, they proposed four types with eight subtypes, including complete celiac trunk, incomplete celiac trunk, absence of the celiac trunk, and celiacomesenteric trunk.

However, all these existing classifications failed to describe the anatomic details or further classify for the absence of the celiac trunk. In these studies, the absence of the celiac trunk was narrowly defined as that each of all its branches arise separately from the abdominal aorta, but excluded any HM trunk + LGA + $\mathrm{SA}, \mathrm{SM}$ trunk + LGA + CHA, or GM trunk + CHA + SA. Our new classification defines the absence of the celiac trunk as that the celiac trunk is not exist, more specifically, there is not such an arterial trunk containing at least two major branches of the celiac trunk. This definition indicates that the absence of the celiac trunk should also include HM trunk + LGA + SA, SM trunk + LGA + CHA, GM trunk + CHA + SA, and any other variant that conforms to the definition, in addition to LGA + CHA + SA + SMA, which thus results in a higher prevalence $(0.76 \%)$ in our study population.

The absence of the celiac trunk was found in 19 patients in our 2,500 patients studied by the MDCT angiography which should be the most samples so far. Here, we first describe the anatomic details of the absence of the celiac trunk and further classify it into different types which have not been seen in the previous literature. By reviewing previous studies and combining with the present findings on the absence of the celiac trunk, we propose a new and useful classification: Type I, LGA + CHA + SA + SM; type II, HM trunk + LGA + SA; type III, SM trunk + LGA + CHA; type IV, GM trunk + CHA + SA; and type V, other type. The new definition and classification are not only helpful for assessing its variant patterns, but also to analyze the potential embryological mechanisms which led to variant formation. 
Tandler [13] stated that four primitive ventral vascular roots (namely LGA root, CHA root, SA root, and SMA root) stem form the abdominal aorta during the early phase of embryogenesis and that these roots are interconnected by a longitudinal anastomosis. During normal development the longitudinal anastomosis is interrupted between roots 3 and 4, thereby resulting in anatomic separation of the celiac trunk from the SMA (Fig. 2a). The misplacement of the longitudinal anastomosis leads to the development of variants in the celiac trunk and SMA. Our new classification on the absence of the celiac trunk enables us to further analyze the embryological mechanisms of its various types.

Type I of the absence of the celiac trunk may be a primitive type, in which LGA, CHA, SA and SMA originate separately from abdominal aorta. This type can be due to abnormal interruptions of the longitudinal anastomosis among all four roots during embryonic development (Fig. 2b). Since the origins of the LGA, CHA and SA approximate at the same level, the up and down position among the three arteries may not be invariant in human embryos. If the root 3 is $\mathrm{CHA}$ and the abnormal interruptions occur among roots 1-3, a type II (HM trunk + LGA + SA) would form (Fig. 2c); if the root 3 is SA, the abnormal interruptions would result a type III (SM trunk+ LGA + CHA) (Fig. 2d); however, only very rarely, the root 3 could be LGA, an extremely unusual type IV (GM trunk + CHA + SA) may occur (Fig. 2e). The type IV may also be attributed to a complete regression or absence of root 1 , with a replaced LGA arising from SMA, while the abnormal interruptions occur among roots $2-4$. Usually, the LGA root is the highest one and the SMA root is the lowest one, they cannot interconnection with each other. Therefore, the type IV is not found in our study, but it is theoretically possible.

The type $V$ can also be explained by abnormal interruptions and persistence of the longitudinal anastomosis, and regression of vascular root and emergence of replaced artery during embryonic development (Fig. 2f). Such as one of our patients, a SM trunk + LGA with the CHA arising from the distal segment of the SMA, may be attributed to a complete regression or absence of root $\mathrm{CHA}$, with a replaced $\mathrm{CHA}$ arising from SMA. The type $\mathrm{V}$ is often presented as a variety of very complex and rare variants. lyori et al [12] reported a special patient, abdominal aortography did not show the celiac trunk, selective arteriography of the SMA showed a dilated and elongated pancreaticoduodenal artery, which led to the gastroduodenal artery, CHA, proper hepatic artery, SA, and other arteries originating from the celiac trunk. It may be attributed to complete regression or congenital absence of the celiac trunk.

The absence of the celiac trunk has important clinical significance in abdominal vascular surgery, such as in liver transplantation [14], acute mesenteric occlusion, laparoscopic surgery and interventional procedures in the upper abdomen, specifically when considering the Appleby procedure [15]. Identification of celiac trunk variants may avoid vascular complications during procedures or lead to target embolization in transcatheter arterial chemoembolization. Although abdominal aortic aneurysm with absence of the celiac trunk is extremely rare, complete preoperative MDCT angiographic visualization of the visceral vessels is needed to achieve vascular control of the upper abdominal aorta in patients with significant intraperitoneal bleeding caused by a ruptured aortic aneurysm [16-18]. The variants of absent celiac trunk may be decisive when planning surgical or radiological upper abdominal procedures. The MDCT is a safe and reliability, convenient and rapid way to assess the celiac trunk variants. We hope that 
the present study would help to minimize complications related to abdominal surgery, including bleeding and necrosis, as well as facilitate better and more accurate radiological interpretation.

The present study has some limitations. First, it was performed retrospectively. Second, it was conducted based on image interpretations performed by means of consensus opinion. Third, the Type IV (GM trunk + $\mathrm{CHA}+\mathrm{SA}$ ) of absence of the celiac trunk was not found in our patients, we need more data to see if it exists. However, the new classification enables us to describe in detail various types of absence of the celiac trunk, and discuss their probable embryological mechanisms.

\section{Conclusions}

In conclusion, we have developed a new definition and classification on the absence of the celiac trunk, and used MDCT angiography to identify its types and prevalence in a large study population. By integrating these variants, we were able to generate hypothetical embryological mechanisms to explain all observed variants. Abnormal interruptions and persistence of the longitudinal anastomosis, and regression of vascular root and emergence of replaced artery could be the embryological mechanisms of the absence of the celiac trunk.

\section{Declarations}

\section{Acknowledgments}

Not applicable.

\section{Abbreviations}

MDCT: Multidetector computed tomography; CMT: Celiomesenteric trunk; CHA: Common hepatic artery; LGA: Left gastric artery; SA: Splenic artery; SMA: Superior mesenteric artery

\section{Authors' contributions}

YW and YM contributed to the study conception and design. YM, WT and BJ contributed data collection. LK contributed quality control of data and algorithms. YM, WT and YW contributed data analysis and interpretation. JZ contributed statistical analysis. YM and WT contributed manuscript writing. YW contributed manuscript editing. All authors read and approved the final manuscript.

\section{Funding}

None.

\section{Availability of data and materials}

The datasets used and/or analyzed during the current study are available from the corresponding author on reasonable request. 


\section{Ethics approval and consent to participate}

The study was approved by the ethics committee of the Daping Hospital of Army Medical University. The need for individual consent was waived by the committee.

\section{Consent for publication}

Not applicable.

\section{Competing interests}

The authors declare that they have no competing interests.

\section{References}

1. Wang Y, Cheng C, Wang L, Li R, Chen JH, Gong SG. Anatomical variations in the origins of the celiac axis and the superior mesenteric artery: MDCT angiographic findings and their probable embryological mechanisms. Eur Radiol. 2014;24:1777-84.

2. Venieratos D, Panagouli E, Lolis E, Tsaraklis A, Skandalakis P. A morphometric study of the celiac trunk and review of the literature. Clin Anat. 2013;26:741-50.

3. Song SY, Chung JW, Yin YH, Jae HJ, Kim HC, Jeon UB, et al. Celiac axis and common hepatic artery variations in 5002 patients: systematic analysis with spiral CT and DSA. Radiology. 2010;255:27888.

4. Iacob N, Sas I, Joseph SC, Pleş H, Miclăuş GD, Matusz P, et al. Anomalous pattern of origin of the left gastric, splenic, and common hepatic arteries arising independently from the abdominal aorta. Rom J Morphol Embryol. 2014;55:1449-53.

5. Yi SQ, Terayama H, Naito M, Hirai S, Alimujang S, Yi N, et al. Absence of the celiac trunk: case report and review of the literature. Clin Anat. 2008;21:283-6.

6. Matusz P, Miclaus GD, Ples H, Tubbs RS, Loukas M. Absence of the celiac trunk: case report using MDCT angiography. Surg Radiol Anat. 2012;34:959-63.

7. Lipshutz B. A composite study of the coeliac axis artery. Ann Surg. 1917;65:159-69.

8. Adachi B. Das Arteriensystem der Japaner, vol II. Kyoto: Maruzen Publishing Co, 1928;20-71.

9. Morita M. Reports and conception of three anomalous cases on the area of the celiac and the superior mesenteric arteries. Igaku Kenkyu. 1935;9:159-72.

10. Michels NA. Blood supply and anatomy of the upper abdominal organs, with a descriptive atlas. Philadelphia, PA: Lippincott, 1955;139-43.

11. Marco-Clement I, Martinez-Barco A, Ahumada N, Simon C, Valderrama JM, Sanudo J, et al. Anatomical variations of the celiac trunk: cadaveric and radiological study. Surg Radiol Anat. 2016;38:501-10. 
12. Iyori K, Horigome M, Yumoto S, Yamadera Y, Saigusa Y, lida F, et al. Aneurysm of the gastroduodenal artery associated with absence of the celiac axis: report of a case. Surg Today. 2004;34:360-2.

13. Tandler J. Über die varietäten der arteria coeliaca und deren entwickelung. Anat Hefte. 1904;25:473500.

14. Ishigami K, Zhang Y, Rayhill S, Katz D, Stolpen A. Does variant hepatic artery anatomy in a liver transplant recipient increase the risk of hepatic artery complications after transplantation? AJR Am J Roentgenol. 2004;183:1577-84.

15. Appleby LH. The coeliac axis in the expansion of the operation for gastric carcinoma. Cancer. 1953;6:704-7.

16. Armstrong PJ, Franklin DP. Superior mesenteric artery branch aneurysm with absence of the celiac trunk. Vascular. 2006;14:109-12.

17. Teng W, Sarfati MR, Mueller MT, Kraiss LW. A ruptured pancreaticoduodenal artery aneurysm repaired by combined endovascular and open techniques. Ann Vasc Surg. 2006;20:792-5.

18. Maier JP, Patetsios P, Moglia RM, Dietz PA. Superior mesenteric artery aneurysm in a patient with congenital absence of the celiac axis. J Am Coll Surg. 2002;195:279.

\section{Tables}

Table 1 Definition and classification for description of absence of the celiac trunk 
Definition and Description

type

Definition

Absence of We defined the absence of the celiac trunk as that the celiac trunk is not exist, more the celiac trunk specifically, there is not such an arterial trunk containing at least two major branches of the celiac trunk.

Type

(abbreviation)

LGA + CHA + LGA, CHA, SA and SMA arising separately from the abdominal aorta

SA + SMA (type

I)

$\mathrm{HM}$ trunk $+\quad$ Hepatomesenteric trunk with LGA and SA arising separately from the abdominal aorta LGA + SA (type

II)

SM trunk $+\quad$ Splenomesenteric trunk with LGA and CHA arising separately from the abdominal aorta $\mathrm{LGA}+\mathrm{CHA}$

(type III)

GM trunk $+\quad$ Gastromesenteric trunk with CHA and SA arising separately from the abdominal aorta $\mathrm{CHA}+\mathrm{SA}$ (type IV)

Other type Any other variant that meets the above definition of the absence of the celiac trunk (type V)

LGA left gastric artery, CHA common hepatic artery, SA splenic artery, SMA superior mesenteric artery

Table 2 Types and percentages of the absence of the celiac trunk $(n=2,500)$ 


\begin{tabular}{llll}
\hline Type & Number & Percentage & $P$ value \\
\hline Normal anatomy* & $2,243 / 2,500$ & $89.72 \%$ & \\
Anatomical variant & $257 / 2,500$ & $10.28 \%$ & \\
$\quad$ Absence of the celiac trunk & $19 / 2,500$ & $0.76 \%$ & 0.524 \\
$\quad$ Sex & & & \\
$\quad$ Male & $15 / 1,811$ & $0.83 \%$ & 0.004 \\
Female & $4 / 689$ & $0.58 \%$ & \\
$\quad$ Type & & & \\
$\quad$ Type I (LGA + CHA + SA + SMA) & $5 / 2,500$ & $0.20 \%$ & \\
$\quad$ Type II (HM trunk + LGA + SA) & $9 / 2,500$ & $0.36 \%$ & \\
$\quad$ Type III (SM trunk + LGA + CHA) & $3 / 2,500$ & $0.12 \%$ & \\
$\quad$ Type IV (GM trunk + CHA + SA) & 0 & 0 & \\
$\quad$ Type V (other type) & $2 / 2,500$ & $0.08 \%$ & \\
Other variant & $238 / 2,500$ & $9.52 \%$ \\
\hline LGA left gastric artery, CHA common hepatic artery, SA splenic artery, SMA superior mesenteric artery. \\
* Normal anatomy refers to hepatogastrosplenic trunk plus SMA & \\
\hline
\end{tabular}

\section{Figures}



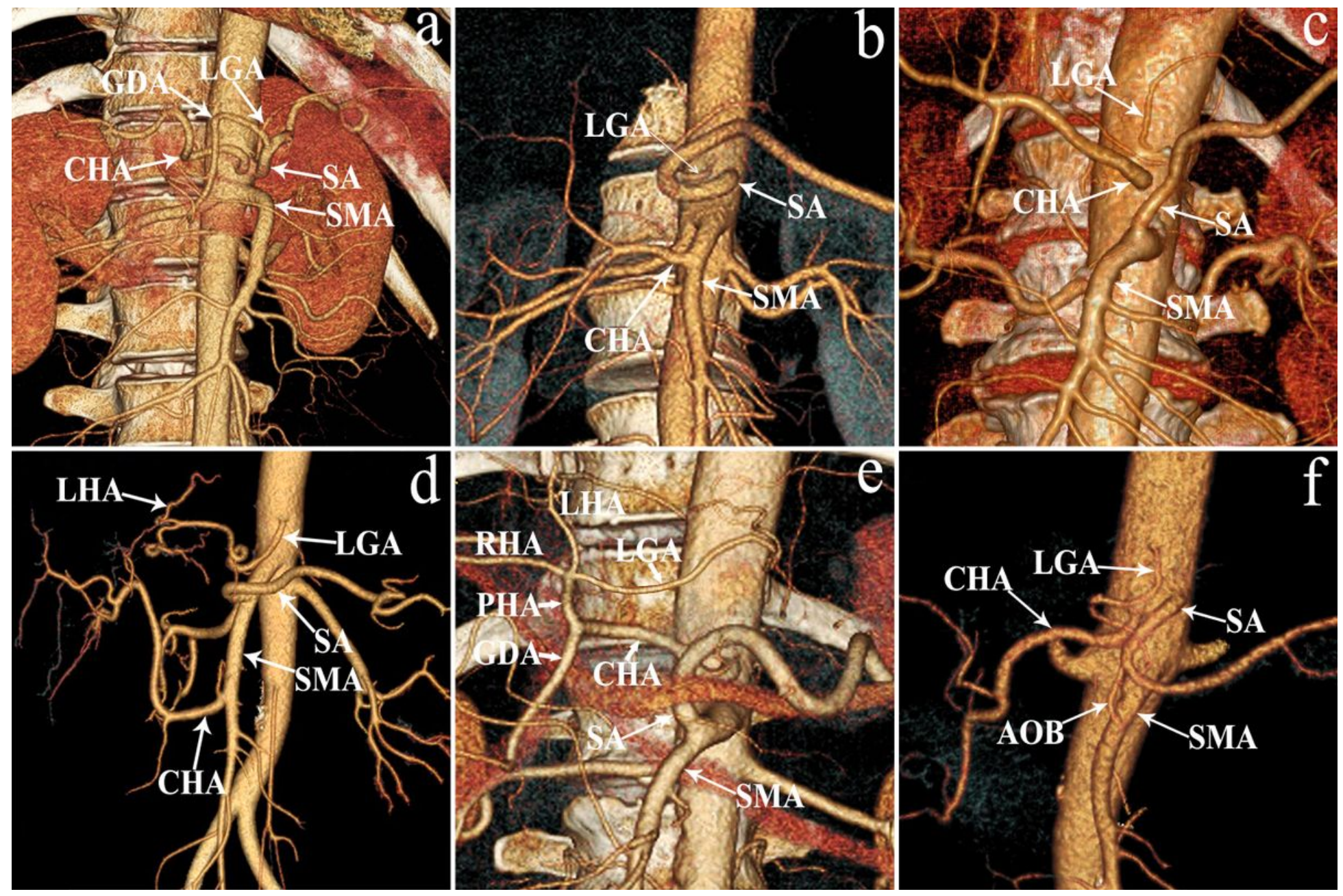

Figure 1

Five types and a few concomitant variants of the absence of the celiac trunk. (a) Type I, the LGA, CHA, SA and SMA originate separately from the abdominal aorta. In addition, the gastroduodenal artery (GDA) arising ectopically from the SA in this patient is a concomitant variant. (b) Type II, the CHA arises ectopically from the SMA (it can also be called hepatomesenteric trunk), and the LGA and SA originate directly from the abdominal aorta, respectively. (c) Type III, the SA arises ectopically from the SMA (it can also be called splenomesenteric trunk), and the LGA and CHA originate directly from the abdominal aorta, respectively. (d) Type V, which is the other type in the new classification. In this patient, MDCT angiography image shows the LGA originating directly from the abdominal aorta, with the CHA and the SA arising ectopically from the SMA at two different sites. Besides, the left hepatic artery (LHA) arising ectopically from the LGA is a concomitant variant. (e) Type V, in another patient, the SA arises ectopically from the SMA and the CHA originates directly from the abdominal aorta, but the LGA arises ectopically from the end of the proper hepatic artery (PHA). (f) Another patient with type I has a concomitant arc of Bühler (AOB) between the CHA and the SMA. 


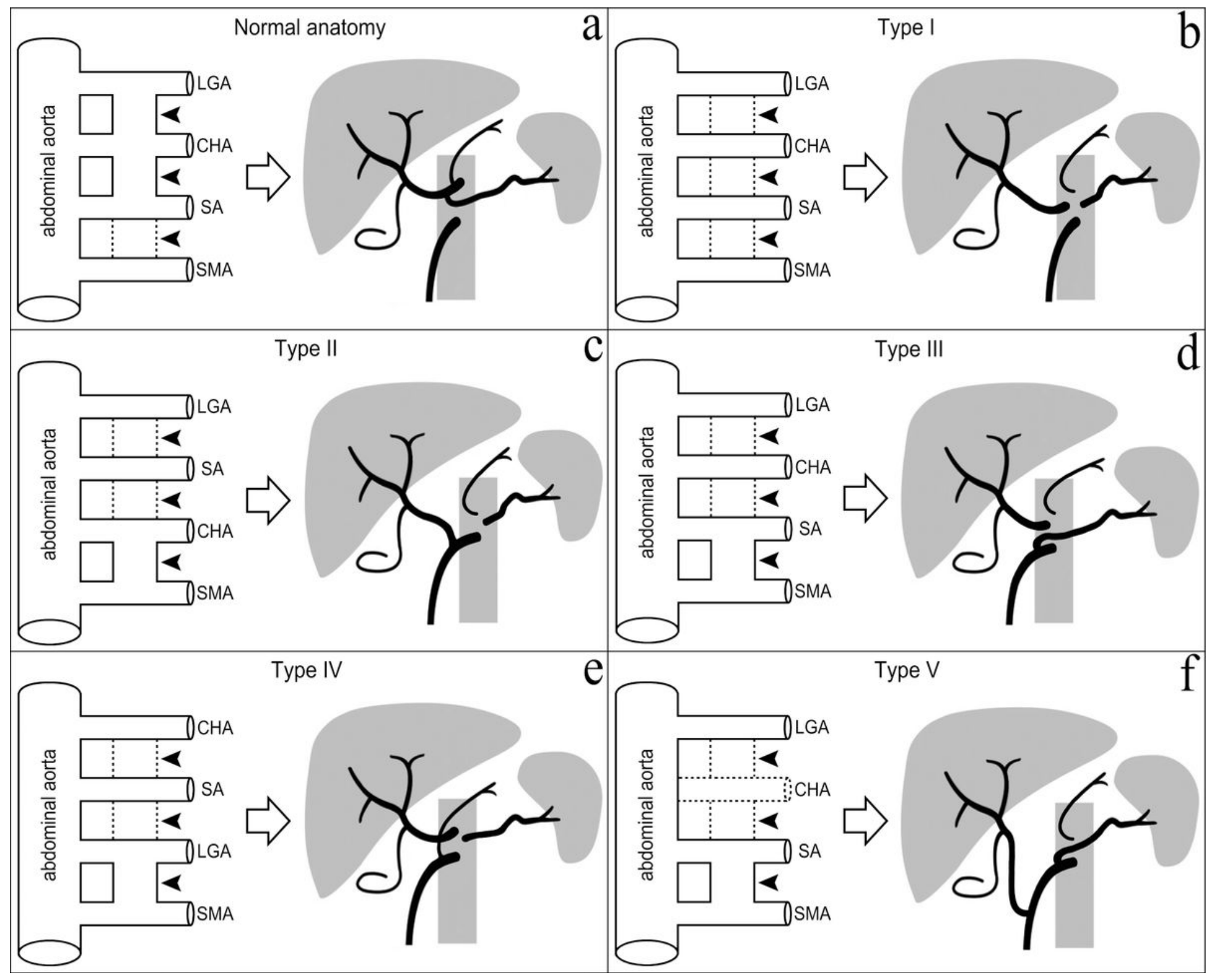

Figure 2

Schematic diagram of embryologic development for the absence of the celiac trunk. The primitive dorsal abdominal aorta is an origin for four ventral vascular roots-LGA, CHA, SA, and SMA. These are joined by a longitudinal anastomosis (arrowheads). (a) When the longitudinal anastomosis is interrupted between roots 3 and 4 (dashed line), the normal configuration of the celiac trunk and SMA can be generated. (b) Abnormal interruptions (dashed line) of the longitudinal anastomosis among all four roots may generate a type I of the absence of the celiac trunk. Since the origins of LGA, CHA and SA approximate at the same level, the up and down position among the three arteries may not be invariant; (c) if the root 3 is $\mathrm{CHA}$ and the abnormal interruptions (dashed line) occur among roots $1-3$, a type II would form; (d) if the root 3 is $\mathrm{SA}$, the abnormal interruptions (dashed line) would result a type III; however, (e) only very rarely, the root 3 could be LGA, an extremely unusual type IV may occur. (f) The type $V$ can also be explained by abnormal interruptions and persistence of the longitudinal anastomosis, and regression of vascular root and emergence of replaced artery during embryonic development. For example, a SM trunk + LGA with the 
$\mathrm{CHA}$ arising from the distal segment of the SMA, it may be attributed to complete regression of root $\mathrm{CHA}$ (dashed line), with a replaced CHA arising from SMA. 